\title{
Supporting Online Dating Decisions with a Prompted Discussion Interface
}

\author{
Douglas Zytko \\ Oakland University \\ Rochester, MI 48309, USA \\ zytko@oakland.edu

\section{Victor Regalado} \\ New Jersey Institute of \\ Technology \\ Newark, NJ 07301, USA \\ vrrs0988@gmail.com

\section{Sukeshini A. Grandhi} \\ Eastern Connecticut State \\ University \\ Willimantic, CT 06226, USA \\ grandhis@easternct.edu
}

\author{
Quentin Jones \\ New Jersey Institute of \\ Technology \\ Newark, NJ 07301, USA \\ qgjones@acm.org
}

\begin{abstract}
Online dating systems are popular tools for pursuing romance, yet first dates between online daters are commonly unenjoyable because of incompatibility signaled through face-to-face conversation. This paper presents the prompted discussion interface, which aims to inform online daters' expectations for face-to-face interactions by prompting them with messaging discussion topics that theoretically spur the expression of attraction-relevant traits. Preliminary findings are presented from a qualitative study $(n=35)$ about user perceptions of the interface.
\end{abstract}

\section{Author Keywords}

Online dating; mediated conversation; messaging;

impression formation; social matching

\section{Introduction}

Online dating systems have become a standard-if not must-have-tool for individuals pursuing romance. Yet despite their popularity, online dating system-use can be frustrating $[1,2,4]$. Research indicates that first dates between online daters are seldom enjoyable [2] because of incompatibility signaled through

conversation $[6,7]$. The research suggests that online daters may have difficulty foreseeing unenjoyable faceto-face conversations because some users behave in ways during mediated conversation (i.e., messaging 


\section{Prompted Discussion Interface

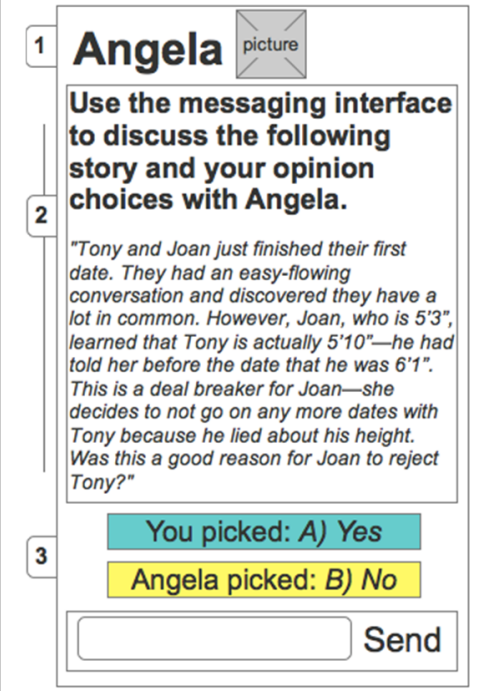

1: The user that one is exchanging messages with.

2: The prompt to discuss the displayed first-date conflict story (see side bar on next page for readable example).

\section{3: The opinion choices} previously picked by both users, which conveys whether they agreed or disagreed on the story. interfaces) that reflect overt impression management and formation strategies, which give little indication of subsequent face-to-face interaction tendencies. For example, some users try to manage attractive impressions with carefully crafted message content, some of which is entirely copy-and-pasted from dating coaches and online forums for dating advice $[4,7]$. This paper presents the design and preliminary qualitative assessment of a messaging interface prototype that aims to support online daters in determining which users they would enjoy face-to-face conversations with.

\section{Design and Study of the Prompted Discussion Interface}

Theories of relationship satisfaction can be used to propose ways that messaging interfaces could better support online daters. Models of marital satisfaction posit that problem-solving discussions (i.e., interactions in which partners are prompted to discuss a topic that they disagree on) are conducive to expression, and therefore assessment, of attraction-relevant traits (e.g., personality) [3]. If this theory extends to potential romantic partners, messaging interfaces that prompt online daters with problem-solving discussion topics may yield interactions online that are similarly enjoyable to future, in-person interactions in which the richer, face-to-face context inherently supports signaling of attraction-relevant traits.

Following a research-through-design approach [5], we designed a messaging interface that prompts two users to discuss a problem-solving discussion topic (see side bar). Problem-solving discussion topics in prior work used marital conflict themes (e.g., a disagreement over when to have a baby) because the research subjects were married couples, but we found such topics inappropriate for online dating because users may not find such scenarios personally relevant. Our interface instead features "first-date conflict stories," or scenarios that depict conflicts that can occur during a first date between potential romantic partners (see side bar on next page for an example).

The prompted discussion interface is the focal point of a mixed methods study that produces 1 ) quantitative data regarding use of the interface by daters, and 2) qualitative data regarding daters' personal reactions to the interface. This paper reports preliminary results from the qualitative component of the study. Given evidence that online daters often introduce their own messaging topics in line with impression management motives $[6,7]$, it is an open question as to whether users would be willing to abandon their regular strategies to follow discussion prompts from the interface. Specifically, this paper explores the following research question: Would online daters be willing to discuss first-date conflict stories if a messaging interface prompted them to do so? Would a disagreement/agreement of opinion on a story with another user change this willingness?

\section{Method}

Focus groups were used to explore the aforementioned research question. Participants were recruited through a Facebook ad that targeted heterosexual singles in New York City for a dating event in which singles would be exposed to an online dating prototype and then meet other singles for short one-on-one conversations or "speed dates." A total of 35 individuals (19 men, 16 women, ages 21-35) participated across four focus group sessions. All focus groups were conducted in a reserved meeting space in New York City. Participants 


\section{First-date conflict story} featured in focus groups: Tony and Joan just finished their first date. They had an easy-flowing conversation and discovered they have a lot in common. However, Joan, who is 5'3", learned that Tony is actually 5'10"he had told her before the date that he was 6'1". This is a deal breaker for Joan-she decides to not go on any more dates with Tony because he lied about his height. Was this a good reason for Joan to reject Tony? (Opinion choices were A) yes, and B) no.)

The story was selected based on results of a Mechanical Turk survey $(n=275)$ that garnered reactions to 34 first-date conflict stories created by the researchers. The selected story procured a $53 \% / 47 \%$ split of opinion (therefore making a disagreement plausible), and most respondents indicated a willingness to discuss the story with potential partners regardless of the opinion choice selected. were separated into male-only and female-only groups to avoid hesitancy to discuss online dating with potential romantic partners present. All but two participants had prior online dating experience.

The focus groups centered on the following scenario: "Imagine you just matched with a man/woman in a popular online dating system. You liked each other's profile page and the online dating system brings you to this messaging interface to start a conversation. What are your reactions?" This scenario was given three times with different messaging interface variations: 1 ) an "open" messaging interface with no discussion prompts (i.e., the typical interface in online dating systems); 2) the prompted discussion interface with verbal instructions to imagine that they and the matched user had disagreed on the respective story; and 3 ) the prompted discussion interface with verbal instructions to imagine that they had agreed on the story. See the side bar for the first-date conflict story shown to participants. All focus groups were voice recorded, transcribed, and subjected to open coding.

\section{Findings}

Male participants were generally enthusiastic about discussing the first-date conflict story because such a prompt alleviated the need for them to decide on their own conversation topics. As Robert explained, "it saves me a bit of work. [...] What should I talk about? With this, I don't have to think about it." Male participants appeared most willing to follow the prompt when the interface conveyed an agreement of opinion with a potential partner. Some of the men interpreted the interface's display of agreement as emphasizing compatibility akin to a "match" (Brian) computed by an algorithm. The conversation prompt, under this interpretation, was seen as the online dating system's way of "helping" male users showcase compatibility.

Bart: "The site is helping me out here, huh? Like 'hey, talk about this thing you have in common. "'

Jack: "I'm guessing you made this [messaging interface] to help guys. You're like making sure we don't say anything stupid by giving us something to talk about with the girl. And [when there's an agreement] it's a positive thing, something we see eye to eye on. [...] Yeah, I would no longer have to worry about what to type."

Female participants were also generally open to discussing the first-date conflict story. Several of them thought such stories would serve as a better starting point for probing deeper into romantic compatibility than the conversation topics that men usually pick. For example, Kristen said: "There are only so many different ways they can start [a messaging conversation] with me. It's either Game of Thrones because that's in my profile, or my looks, or just 'hey how are you.' [...] I'd get a lot more information about us as a potential couple with these [first-date conflict stories]." See the sidebar on the next page for an additional example from Jo.

However, some of the women were hesitant to discuss stories that culminated in disagreements with other users. This stemmed from prior online dating experiences in which men reacted poorly to disagreements that arose during messaging. While most female participants indicated they would be willing to discuss disagreements in the interface, they 
Jo: "Yes, yes, yes. Messaging is my least favorite part of online dating. I could show you some of the messages I get. They are just so bad and you can't get a conversation started from any of that. I think it would be a lot of fun to talk about these [stories]. [...] I bet it would save me from going on a lot of bad dates with guys that can't talk to save their life."

Cindy: "This one guy, right. He was talking about some restaurant we both know and I said it's pretty overrated. A tourist spot. He freaked out at me! Saying I have no taste and all that. Well excuse me for having a different opinion. [...] I wouldn't want to get into a lot of those conversations. So me, personally, I'm fine with the guy picking a different opinion choice from me. Would he be okay though? If you could somehow check that, to make sure the guy wouldn't freak out that I disagreed, this would probably be a great, great feature to have." were concerned about negative reactions from their conversation partners (see quote from Cindy).

\section{Discussion and Next Steps}

This paper proposed the prompted discussion interface as a tool for helping online daters determine who they would enjoy conversation with on face-to-face dates. Preliminary qualitative insight suggests that online daters would generally be willing to engage with discussion topics prompted by the interface. However, there is also indication that the interface, in its current iteration, may misinform online daters' expectations of subsequent face-to-face interactions. For one, some male participants interpreted a displayed agreement of opinion on a story as a "match" with a potential partner. This was not intended in design and can mislead users into expecting romantic compatibility on a face-to-face date. In addition, some female participants said they would be cautious about discussing disagreements because they assumed men would react negatively. This may incur psychological effects during messaging interactions that can mislead expectations for subsequent interactions. These findings suggest some modifications to the interface, such as clarifying to users that displayed opinion choices are not intended to signal a romantic "match" or incite an argument.

Ongoing research efforts involve a quantitative assessment of the interface in which daters use the interface to converse with potential romantic partners before meeting them face-to-face to confirm their online assessments.

\section{Acknowledgements}

This research is partially supported by a grant from the National Science Foundation 1422696.

\section{References}

1. E. J. Finkel, P. W. Eastwick, B. R. Karney, H. T. Reis, and S. Sprecher. 2012. Online Dating: A critical analysis from the perspective of psychological science. Psychological Science in the Public Interest 13, 1: 3-66.

2. Jeana H Frost, Zoe Chance, Michael I Norton, and Dan Ariely. 2008. People are experience goods: Improving online dating with virtual dates. Journal of Interactive Marketing 22, 1: 51-61.

3. Benjamin R Karney and Thomas N Bradbury. 1995. The longitudinal course of marital quality and stability: A review of theory, methods, and research. Psychological Bulletin 118, 1: 3-34.

4. Christina Masden and W Keith Edwards. 2015. Understanding the role of community in online dating. In CHI Proceedings, 535-544.

5. John Zimmerman, Jodi Forlizzi, and Shelley Evenson. 2007. Research through design as a method for interaction design research in HCI. In CHI Proceedings, 493-502.

6. Douglas Zytko, Sukeshini A Grandhi, and Quentin Jones. 2014. Impression management struggles in online dating. In Proceedings of the 18th International Conference on Supporting Group Work, 53-62.

7. Douglas Zytko, Sukeshini A Grandhi, and Quentin Jones. 2016. The coaches said...what?: Analysis of online dating strategies recommended by dating coaches. In Proceedings of the 19th International Conference on Supporting Group Work, 385-397. 